\title{
Investigation of an Eight-Week Neuromuscular Training Intervention on Biomechanical Parameters of the Lower Quarter in Adolescent Female Soccer Players
}

\author{
Craig Parker, Kevin Robinson, Trent Nessler, Pat Sells, Kayla Lydon \\ School of Physical Therapy, Belmont University, Nashville, USA \\ Email: craig.parker@adjuncts.belmont.edu
}

How to cite this paper: Parker, C., Robinson, K., Nessler, T., Sells, P. and Lydon, K. (2022) Investigation of an Eight-Week Neuromuscular Training Intervention on Biomechanical Parameters of the Lower Quarter in Adolescent Female Soccer Players. Open Access Library Journal, 9: e8239.

https://doi.org/10.4236/oalib.1108239

Received: November 29, 2021

Accepted: January 15, 2022

Published: January 18, 2022

Copyright () 2022 by author(s) and Open Access Library Inc.

This work is licensed under the Creative Commons Attribution International License (CC BY 4.0).

http://creativecommons.org/licenses/by/4.0/

\section{Open Access}

\begin{abstract}
Purpose: Mounting evidence supports the implementation of neuromuscular training (NMT) interventions to improve biomechanical profiles for prevention of musculoskeletal injury in dynamic pivoting athletes. Research has demonstrated there is a clear link between functional movement behavior and vulnerability to injury. However, there is limited research examining the capacity of NMT to positively influence pathomechanical movement behavior. This investigation assessed the strength, balance, and functional biomechanics of uninjured adolescent female athletes following an eight-week NMT intervention, which was conducted in order to expand upon research aimed at injury prevention in the lower quarter. Methods: 37 female soccer players ages 10 - 15 participated. Hip strength was measured with hand dynamometry, and single-leg stance modified balance $\left(\mathrm{SLS}^{\mathrm{M}}\right)$ was measured in multiple conditions. A 3-Dimensional Dynamic Movement Assessment (3D-DMA) system assessed lower quarter joint excursion during select functional loading tasks. Participants completed an 8-week, 16-session NMT intervention followed by repeated measurements. Results: Following the intervention, significant improvements were found in: hip abduction strength bilaterally ( $p=$ $0.000)$, hip extension strength bilaterally $(p=0.000), \mathrm{SLS}^{\mathrm{M}}$ in eyes-closed condition bilaterally $(p=0.000)$, and DMA functional outcomes in the Full Squat Test $(p=0.019)$, Step-Up Test $(p=0.007)$, Single-Leg Squat Test $(p=$ $0.000)$, and Single-Leg Hop Test $(p=0.000)$. Conclusions: These data indicate an 8-week NMT intervention is sufficient to elicit positive neuromuscular adaptations in the lower quarter associated with pathomechanical loading patterns. Such adaptations support improved function across a diversity of complex sport-related movements. More research is needed to further devel-
\end{abstract}


op the efficacy of NMT interventions in various at-risk populations across a

range of time scales.

\section{Subject Areas}

Biophysics, Kinesiology

\section{Keywords}

Neuromuscular Training, Motor Control, Injury Prevention, Adolescent

Female Soccer Players

\section{Introduction}

Athletic participation across the lifespan is an evolving phenomenon with increasing physical demands placed on the body as a greater amount of time is invested in both training and competition. A natural consequence of this reality is a substantial accumulation of serious injuries in athletes with varying degrees of complexity and consequence, depending on the nature of trauma that occurs. The development and implementation of interventional programs in parallel with these assessments can effectively attenuate risk for musculoskeletal trauma, allowing for a comprehensive patient/athlete care model. Given the paucity of interventional studies in the literature that investigate specific, high-risk movement behaviors related to injury in a pre/post-test research design, there is a clear need for more inquiry into this area of study.

While virtually all injuries are of interest to the sports medicine clinician, trauma to the anterior cruciate ligament (ACL) of the knee has taken center stage alongside concussion in the field of sports medicine clinical research over the last several decades. A variety of factors have contributed to this development, not least of which including the highly concerning rate of traumatic incidence occurring in dynamic pivoting athletes, and the range of consequences that inevitably follow such injury. ACL rupture is one of the most common major adversities that can occur in sports participation, especially in lower quarter pivoting athletes, with over 125,000 incidents per year documented in the United States alone [1].

Soccer (world football) is one of the most popular sports in the world and has been a domain of increasing interest in medical research over recent years due to the remarkably high incidence of ACL injury documented in this participating population, with developing females recognized as the highest risk of individuals for sustaining this injury [2]. Remarkably, epidemiological data indicate that females are at least twice more likely to sustain an ACL rupture than their male counterparts [3].

The cost associated with serious musculoskeletal trauma is multi-layered, involving physical, psychological, social, and financial consequences that require immense attention and investment of resources. Economically, the long-term 
financial cost of an ACL tear has been estimated to be $\$ 38,121$ for ACL reconstruction and $\$ 88,538$ for rehabilitation without reconstruction [4]. These values include the cost of the initial reconstruction and rehabilitation, along with future management of anticipated complications, such as osteoarthritis, which may require arthroscopy and/or total knee replacement procedures in addition to general orthopedic rehabilitation interventions over time [4]. Over recent decades the incidence of ACL reconstruction among 13- through 17-year-olds has continued to increase in both genders, with disproportionately higher frequency noted in females when compared to males [5].

In addition to financial considerations, the tremendous psychological burden imposed on injured athletes is substantial, detrimentally affecting both sport and quality of daily life. The incidence of depression has been reported as high as $45 \%$ in those suffering from orthopedic trauma [6] and $42 \%$ in patients undergoing ACL reconstruction (ACLR) specifically [7]. Compounding this phenomenon, comorbidities such as depression have been associated with poor clinical outcomes after ACLR [8], warranting increased attention towards a holistic approach to treatment and rehabilitation with a biopsychosocial lens. The opportunity cost of ACLR is also considerable, with athletes reported to miss an average of 10 - 15 months of play following injury [9]. Previous studies have reported that while $76 \%$ of males and $67 \%$ of females return to their sport, less than $50 \%$ of those who suffer an ACL injury return to their previous level of performance [10] [11]. Furthermore, multiple authors report that $12 \%-20 \%$ of athletes who return to full sport participation undergo another ACL injury, with the small majority of subsequent ACLR procedures required on the opposite limb [9] [12].

As our understanding of movement behavior and injury evolves over time, so too does our capacity to utilize functional movement assessments that are both sensitive and increasingly comprehensive in nature. Such assessments are necessary in order to accurately reflect the complexities of human movement behavior that are expressed in challenging and unpredictable athletic environments. The use of such tools is highly informative in both the initial assessment of any individual, as well as the measurement of progress as any given rehabilitative training program is employed. The quantification of the extent to which neuromuscular training (NMT) programs can influence such behavior is a core intention of the present research study.

\section{Pathomechanics of the $A C L$}

The ACL functionally exists to oppose excessive anterior translation of the femur on the tibia, in addition to extremes of knee extension, varus, valgus, and axial rotation [10]. The most common mechanism of injury (MOI) to this structure is a large valgus-producing force dispersed through the knee complex, involving an excess of femoral adduction and internal rotation while the foot is planted in a closed-kinetic-chain (CKC) position [10] [13]. Additional MOIs previously documented in the literature include the following: stiff landing postures characterized by suboptimal hip and knee flexion angles during the landing 
phase of airborne maneuvers, rapid deceleration during a sprint or change of direction (COD), and severe hyperextension moments of the knee during CKC loading [10] [13]. The common theme emerging among all MOIs described in the literature is an excessive reliance on passive anatomical structures of the lower quarter to absorb the distribution of forces. This phenomenon unfolds concurrently with insufficient neuromuscular activation and neuromotor recruitment resulting in poor attenuation of force loads throughout the body.

Risk factors for ACL injury can be divided into categories based on both their quality as intrinsic vs. extrinsic in nature, as well as their ability to be influenced with any given intervention. Those in the present medical landscape which cannot be changed to a significant degree are termed non-modifiable risk factors and include anatomic, hormonal, and genetic factors [14]. Extrinsic risk factors are those that are outside of the control of the individual such as the playing surface and the weather conditions and are likewise non-modifiable. By contrast, modifiable risk factors intrinsic to the individual such as neuromuscular control, functional mobility, and biomechanics are susceptible to influence with NMT interventions [14].

It follows that amongst our greatest opportunities to affect the lives of athletes from both a prophylactic and rehabilitative perspective are derived from the knowledge of such modifiable risk factors, wherein interventional methods can be constructed to elicit positive adaptations over time. Interventions of this nature have shown great promise in reducing the incidence rates of ACL compromise as well as other musculoskeletal trauma associated with the dynamic pivoting athlete [15]. A constructive view of the landscape wherein such changes can be produced is afforded upon deeper examination of the key nuances which characterize the so-called "stiff" landing strategy and the dynamic valgus collapse in contrast.

First, a stiff landing is characterized by a rigid posture of the trunk and lower quarter upon high-velocity CKC deceleration from an airborne position, wherein a strong quadriceps contraction in the knee concurrent with a relatively weak reciprocal contraction of the gluteal and hamstring musculature results in a net anterior translatory shearing moment of the femur on the tibia, resulting in a significant distribution of provocative force to the ACL (see Image 1). Conversely, a "soft landing" strategy involves a relative increase in hip and knee flexion range of motion excursion by comparison during the moment of landing and is associated with increased recruitment of active neuromuscular structures of the proximal hip and posterior knee complex in sequence, allowing for a more optimal attenuation of load distribution throughout the chain of musculoskeletal tissue [16].

Second, the mechanics of the dynamic valgus collapse present uniquely distinguishing characteristics which warrant careful consideration in this particular landscape. This phenomenon is observed upon loading either or both lower extremities in the CKC during a squatting, landing, or similar COD moment, wherein 
femoral adduction and internal rotation drive the knee towards midline producing a valgus moment at the tibiofemoral complex (see Image 2). Additional biomechanical compensations and substitutions often presenting amidst this event may include tibial external rotation, excessive foot pronation, and/or a lateral compensatory trunk lean. The resultant forces produced from these loading moments create a high degree of torsional stress and shear on the ACL, in addition to medial knee capsular and ligamentous stretching, and compression of the lateral compartment of the knee. These additional force vectors are explanatory of how additional structures such as the cartilage and/or medial collateral ligament are often damaged or compromised entirely in the event of ACL rupture.

A larger knee valgus angle during CKC loading is characteristic of a movement strategy in which there is insufficient employment of the proximal hip stabilizers to decelerate the body's center of mass [17]. Again, compared to males, females typically display a greater non-sagittal plane movement at the hip during running and walking [17] and a larger valgus moment at the knee in the early deceleration phase of closed-chain actions of the lower quarter [18].

The stiff landing strategy and the dynamic valgus collapse are the most common mechanisms of injury for non-contact ACL tears [1]. Non-contact injuries occur in the absence of impact from an opposing athlete [15] [19] and comprise

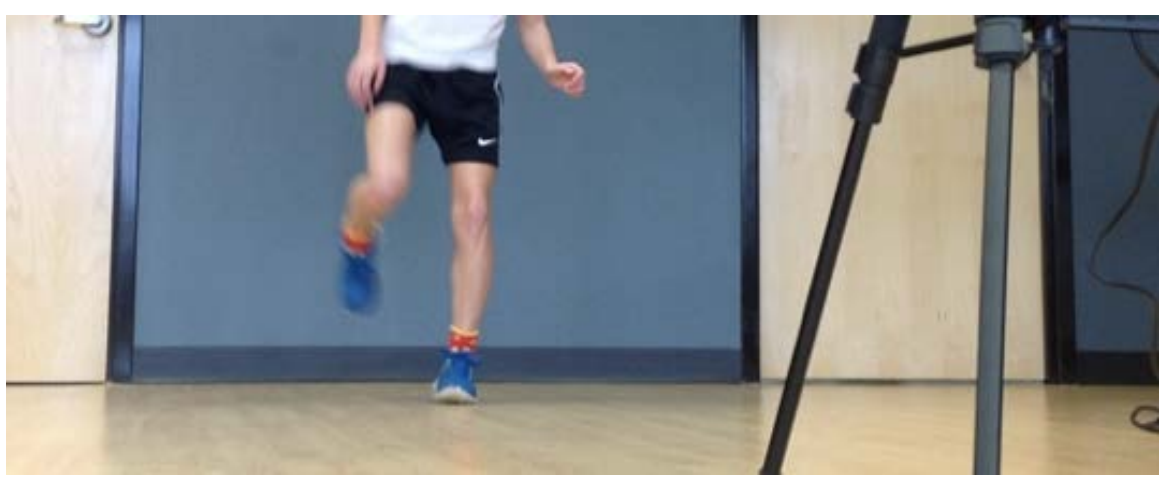

Image 1. A stiff landing pattern presenting in the left lower extremity of a participant in the investigation shown at pre-test interval during the single-leg hop assessment.

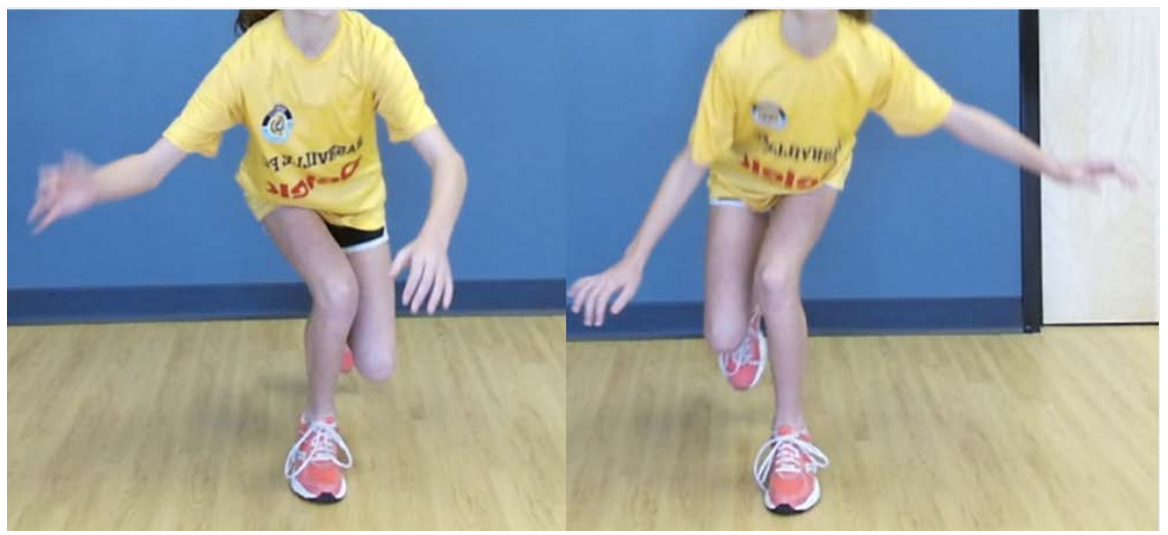

Image 2. The dynamic valgus collapse presenting right and left lower extremities at pre-test interval with a participant in the investigation during the single-leg hop assessment. 
greater than $70 \%$ of all ACL injuries reported [1]. The absence of contact by another during the moment of rupture implies that such injuries occur due to an insufficient ability of the individual to control their body's excursion through space during a highly demanding physical loading moment. The modifiable risk factors described above are primary contributors to these incidents and therefore warrant deliberate attention in both injured and non-injured athletes.

Notably, the soft landing strategy described above is subsequently associated with a relative decrease in valgus loading stresses through knee complex [16]. Insufficient neuromuscular control of the hip musculature especially has been strongly linked to disproportionate knee valgus and stiff landing strategies during CKC loading moments [16] [17]. Females who demonstrate larger knee valgus angles have been shown to have lower hip extensor moments and less energy absorption at the hip during deceleration of drop-jump testing [17]. Conversely, during the same drop-jump test, females who rely more on the hip extensor musculature to absorb impact exhibit fewer knee valgus angles and a 53\% reduction in gross knee valgus movement [17].

Although there is debate in the literature as to whether greater risk of injury is associated more with valgus loading or with stiff landing, both are widely acknowledged as problematic behaviors that expose individuals to elevated risk of trauma [17]. What remains consistent is that females often exhibit a biomechanical profile characterized by high-risk behaviors associated with motor control impairments, which place them at greater risk for ACL injury relative to their male counterparts.

World football is one sport in which ACL injury occurs with remarkable incidence, making it of particular interest to professionals in sports medicine and clinical rehabilitation due to high levels of global participation across the lifespan and the close similarities that the game shares with other highly attended dynamic pivoting sports such as basketball, tennis, volleyball, lacrosse, and American football, each of which also involving large volumes of high-velocity COD moments in the lower quarter in both training and competitive action. There is a degree of generalizability therefore that can be inferred from outcomes produced and observed in the population selected for the present investigation. In general, muscle weakness, suboptimal motor sequencing, and insufficient proprioceptive sensitivity are commonly addressed factors during NMT interventions. Such programs are intended to develop proficiency and resilience in each of these dimensions in effort to facilitate and support optimal movement behavior across a diversity of functional sporting demands and to positively influence the high-risk movement patterns summarized in this manuscript which are associated with increased vulnerability to injury.

In addition to the biomechanical factors summarized here and in previous literature, a substantial amount of evidence supports the notion that psychological factors have a critical role to play in injury incidence and performance capacity. These factors include but are not limited to: subjective fear of re-injury, the sen- 
sation of "not trusting" the knee, and poor function of the knee [10] [11] [20]. An additional hypothesis that is not formally explored in this manuscript is that intensive NMT interventions addressing and promoting the health of multiple dimensions of knee function will serve as a platform to build confidence for the athletes. For these reasons, in addition to the known increase in tissue vulnerability to trauma following injury, the present authors support the premise that a multifactorial, criterion-based return-to-sport approach should be adopted for clinicians in sports medicine and athletic training environments, based on shared decision-making within a broad biopsychosocial framework.

There is a clear need for further research on interventional methodologies which can contribute to the widespread adoption, implementation, and adherence to NMT programs for injury prevention in vulnerable communities, and to adopt a paradigm to translate promising evidence into effective large-scale population-based injury prevention programs [5]. The purpose of the present investigation was to evaluate the lower quarter biomechanics and associated neuromuscular control of adolescent female soccer athletes during select functional movements using a 3-dimensional motion analysis system before and after an 8-week NMT intervention, including a quantification of the subjects' strength and unilateral balance before and after the intervention. The training intervention focused on proximal hip strength, dynamic balance, motor sequencing, functional mobility and COD strategies in the athletes. We hypothesize that the NMT intervention will improve the biomechanical profiles of the participants when comparing pre- and post-testing measures.

\section{Methods}

\section{Subjects}

The subjects included a total of 54 female athletes ages 10 - 15 having an average height of $159.96 \mathrm{~cm}$ and an average weight of $52.24 \mathrm{~kg}$, recruited from a local youth soccer club. The subjects agreed to participate in a six-session training intervention that spanned 8 weeks in duration. In total, 38 athletes consented to undergo testing and motion analysis before and after the training intervention, with one subject dropping out midway through the intervention after suffering from a concussion unrelated to soccer, yielding a total of 37 subjects for statistical analysis. On average, the 37 athletes attended 5.5 out of the 6 sessions, yielding compliance of $91.67 \%$.

\section{Instrumentation}

A Detecto 439 Eye-Level Beam Scale with Height Rod was utilized for measuring subjects' biometrics, including weight in $1 / 4$ pound increments and height in inches and centimeters. The scale allows for measurement up to 400 pounds with a readability of $4 \mathrm{oz}$ [21]; A Lafayette Hand-Held Dynamometer (Manual Muscle Tester Model 01165) was used as a strength measurement tool to quantify force output in kilograms [22]. The 3-Dimensional Dynamic Movement Assessment ${ }^{\mathrm{TM}}$ (3D-DMA) motion analysis software with integrated Microsoft Kinect v2 sensors 
was utilized to record biomechanics with sensors at the pelvis, left and right thighs, left and right legs, and left and right feet (see procedures for anatomical landmarks). A Dell laptop computer was used for data collection and recording.

The Microsoft Kinect sensors actively emit infrared light while utilizing an infrared-sensitive camera to create a depth image at 30 frames per second [23]. The researchers monitored sensor activity and signal in real time during the 3D-DMA data collection. A software package that works with the Kinect, The Microsoft Kinect SDK, fits a 20-point skeletal model onto the image with the points located at the joints [21]. Weber et al. compared the Kinect sensor with data from a Vicon Optical camera system and found that the Kinect system was capable of assessing motion of walking and running (on a treadmill) with acceptable accuracy when analyzing joint angles of the human body in the frontal plane [24].

\section{Procedures}

All procedures were approved by the Belmont University Institutional Review Board. Each participant and guardian were given a child assent form and a parental informed consent for review and signature prior to enrolling in the study, following which participants were assigned an identification number. Once this process was completed, the participant's height and weight were recorded. Immediately after, an assessment of bilateral hip abduction and extension strength was completed using the Lafayette Hand-Held Dynamometer. The administrator held the hand dynamometer between his/her hand and the subject's distal femur at distinct anatomical landmarks with appropriate spatial orientation specific to each of the muscle groups tested. The administrator then instructed the subject to exert as much force as possible against the device for 3 separate attempts, allowing for rests between each, as needed. The maximum forces were recorded and averaged.

The participants were then asked to perform a modified single-leg stance $\left(\mathrm{SLS}^{\mathrm{M}}\right)$ balance assessment by standing on one leg at a time with the hip and knee of the weight-bearing side each in a position of slight flexion (approximately 15 - 30 degrees). The rationale for the modified balance conditions stated was to deliberately remove the knee from a closed-packed position in full extension, in an attempt to accurately simulate the demands of on-field athletic participation. The length of time the subject could maintain balance on each lower extremity was assessed using a stopwatch. The subjects were then asked to perform the same balance task on each leg with the additional condition of maintaining full closure of both eyes through the duration of each test. The subjects were again timed using a stopwatch, with an upper ceiling for the assessment placed at 30 seconds. If the subject completed the 30 seconds without faltering, they were given a score of 30 seconds. For each of the balance tests, scores were recorded as the number of seconds each athlete was able to maintain balance on the limb, with the following cutoff criteria for terminating the assessment: 1) allowing the non-weight bearing limb to touch the floor, 2) changing the original position of the foot in any observable manner or jumping to regain balance, 3 ) deviation of postural 
orientation to an extent that a fall was anticipated (verbal and/or tactile interference required from examiner to prevent potential injury). A fourth cutoff criterion was adopted for the eyes-closed condition of the balance test to include opening of the eyes to any extent that may provide visuospatial reorientation.

After all the above was completed, the participants were then taken through the 3D-DMA assessment. The subjects positioned themselves 5 - 7 feet in front of the Microsoft Kinect sensor. The 3D-DMA software then identified the subject's center of gravity with the camera. The subjects were asked to perform a series of tests in which the 3D-DMA cameras assessed their limb kinematics in the frontal and sagittal planes. The subjects were first asked to perform 20 full-body squats. Each repetition was scored with a possible 3 points, allowing for 60 total points. After the squats, the subject performed the following: 10 step-ups bilaterally, 10 single-leg squats bilaterally, and 10 single-leg jumps bilaterally. Each of these unilateral assessments was scored at a maximum of 3 points per repetition, producing an overall maximum potential score of 240 .

Once the subjects completed testing, they participated in a NMT intervention that included 6 sessions over 8 weeks. These interventional training sessions were implemented on a progressive basis with 5 tiers of focus: isolated strength, dynamic balance, neuromuscular re-education (NMRE), multiplanar motor control, and functional mobility/recovery. Isolated strengthening activities were introduced first which focused predominantly on proximal hip, core, and posterior chain targets including the gluteal muscles, smaller hip rotators, hip abductors, knee flexors, spinal stabilizers, and ankle stabilizers. A fatigue model was employed for prescriptive purposes which dictated that the participants complete each strengthening activity up until the onset of healthy muscle fatigue (not to failure), with the condition that neither pain emerged nor loss of sufficient technical form was observed. Elastic band resistance was utilized with placement around the distal femurs bilaterally throughout each of these activities.

Dynamic balance training consisted of various single-leg weight-bearing activities performed in multiple positions and at various depths of hip and knee flexion with both eyes open and eyes closed training conditions. NMRE activities were characterized by extended submaximal holds performed in a range of open and closed chain postures, held for periods of 30 seconds to 3 minutes (activity, tolerance, and progress dependent). Multiplanar motor control strategies were incorporated first during slow, developmental sequencing tasks and eventually progressed into high-speed, plyometric movements across a variety of sport-specific actions including jumping, landing, rapid COD actions, and oscillating unilateral weight-bearing maneuvers. Functional mobility and recovery activities were completed in all primary lower quarter muscle groups as warm-up, cool down, and active rest intervals to facilitate flexibility, preparedness, and tissue recovery. Dynamic stretches were utilized predominantly in earlier stages of a given session, whereas prolonged stretches were integrated at the end of sessions.

Participants were instructed to withhold from any activity which produced 
pain, and were advised only to move through ranges of motion which could be sufficiently controlled with appropriate biomechanical technique as instructed. Complex activities requiring greater skill levels were integrated progressively as proficiency developed to a satisfactory level, and the athletes were instructed on methods to incorporate training techniques on a principle-driven basis into regular team warm-ups for both physical and cognitive/conceptual reinforcement.

The participants were each provided with additional instruction in-home training activities to be completed 2 - 4 times per week on days that training and competition were not already scheduled. In parallel with the direct contact portion of the training intervention, the home training program activities were structured in a progressive manner, advancing from isolated strengthening and introductory balance to training techniques that required dynamic movements of increasing complexity. These activities were prescribed following the first training session, and recommended to be completed at least once per day on days in which team training or match competition were not already occurring. After all aspects of the intervention were completed, the subjects returned to the laboratory and repeated the post-intervention testing in the same order as described above in the pre-test assessment.

\section{Results}

\section{Hip Abduction and Extension Strength}

A paired samples t-test showed a significant difference in time bilaterally for hip abduction strength $(p=0.000)$ and hip extension strength $(p=0.000)$ between pre-test and post-test strength measures (Graph 1 ). Left hip abduction strength improved from a mean of $8.94 \mathrm{~kg}$ pre-training to $23.05 \mathrm{~kg}$ post-training, and right hip abduction improved from 8.64 to $23.23 \mathrm{~kg}$. Mean left hip extension improved from 7.09 to $15.04 \mathrm{~kg}$, and mean right hip extension improved from 6.86 to 17.68 $\mathrm{kg}$. The data were normally distributed and no significant interactions between sides were identified for hip abduction or hip extension strength measures.

\section{Single-Leg Stance ${ }^{M}$ Balance, Vision Unobstructed:}

When assessing SLS ${ }^{\mathrm{M}}$ balance with eyes open, a paired samples t-test yielded no significant differences in time $(p=0.160)$ for either extremity, pre-test to post-test. SLS ${ }^{\mathrm{M}}$ measures with eyes open on the left lower extremity improved by a trivial margin from 29.46 seconds pre-training to 30 seconds post-training. Given that there was a 30-second maximum cutoff for balance assessment, there was no significant change for balance with eyes open of the right lower extremity as this was found to be 30 seconds at both pre-testing and post-testing. No significant interactions between sides were identified.

\section{Single-Leg Stance ${ }^{M}$ Balance, Vision Occluded}

A paired samples t-test showed a significant difference in time $(p=0.000)$ bilaterally, pre-test and post-test, for SLS ${ }^{\mathrm{M}}$ balance with vision occluded (Graph 2). Measures of the left lower extremity improved from a mean of 16.0 seconds pre-training to 26.05 seconds post-training, while right extremity measures 
Hip Abduction \& Extension Strength Outcomes, Pre - vs. Post-training

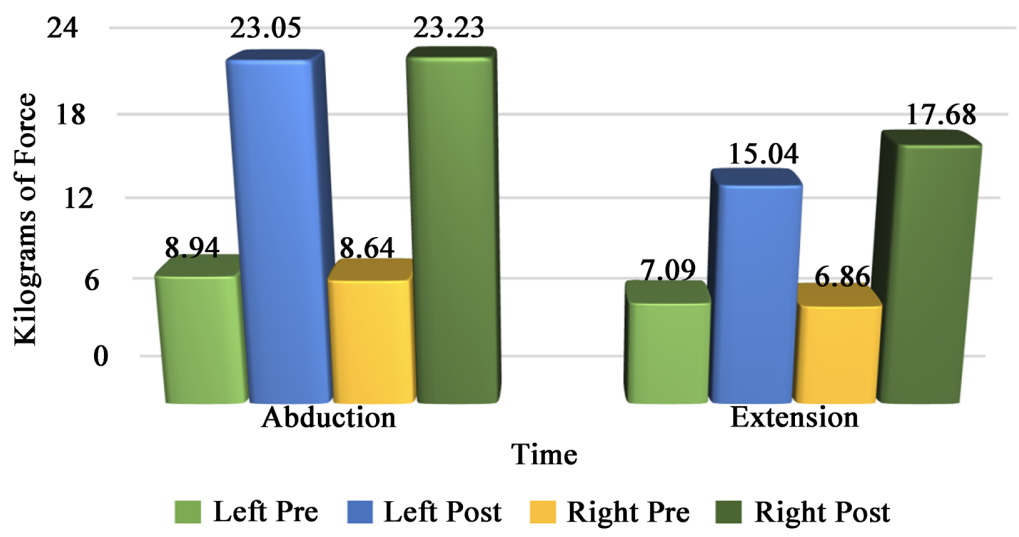

Graph 1. Hip strength measures (kilograms of force exerted) depicted along vertical axis, across Time 1 (pre-test interval) and Time 2 (post-test interval) dimensions on the horizontal axis. Hip abduction measures are depicted on the left and hip extension measures are on the right. For both measures, left lower extremity pre-test shown in green vs. post-test in blue; right lower extremity pre-test shown in yellow vs. post-test in red.

Single-Leg Stance ${ }^{\mathrm{M}}$ Balance with Vision Occluded, Pre- vs. Post-training Outcomes

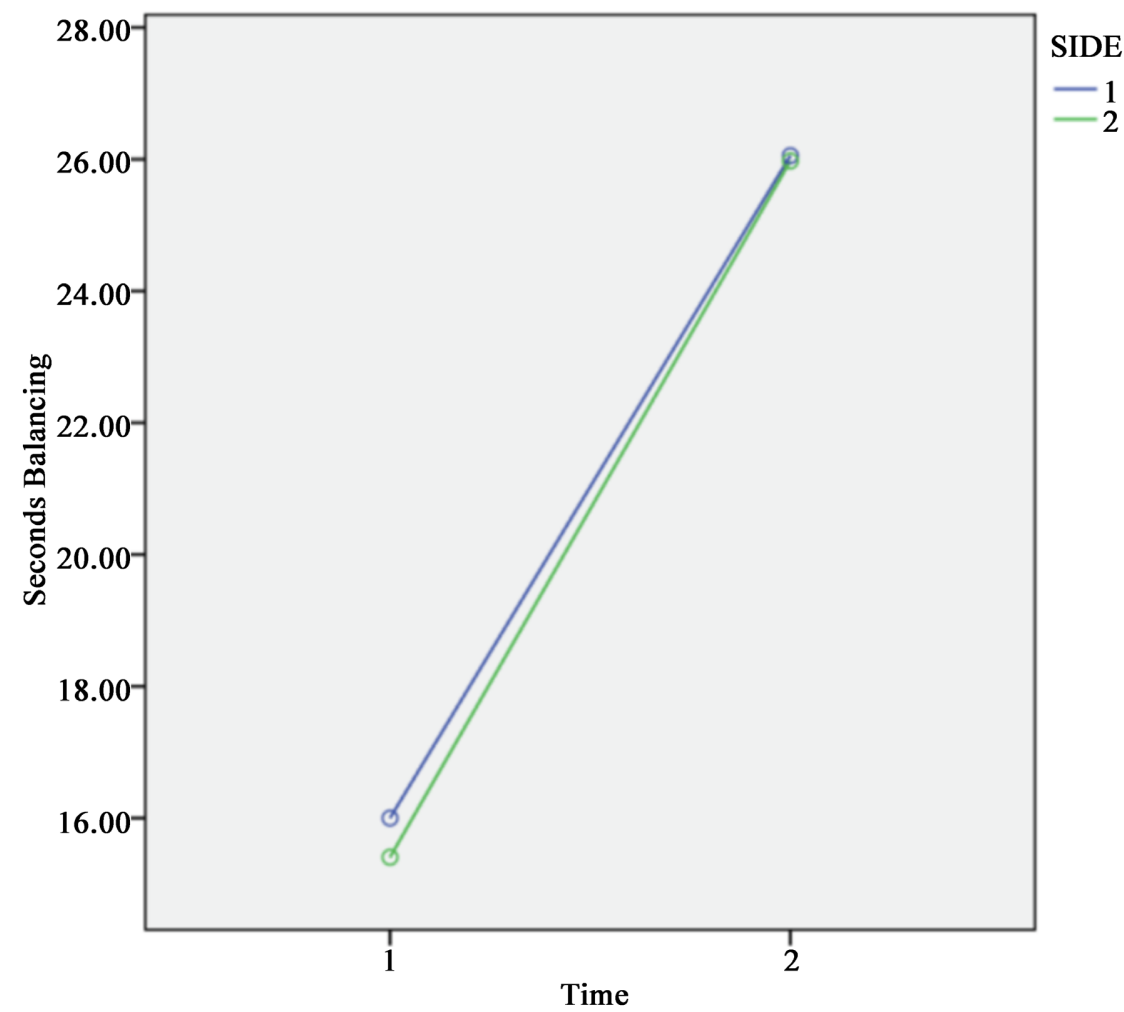

Graph 2. Single-leg stance ${ }^{\mathrm{M}}$ balance measures (seconds held) depicted along vertical axis, across Time 1 (pre-test interval) and Time 2 (post-test interval) dimensions on the horizontal axis. Left lower extremity shown as blue line, right lower extremity shown as green line.

improved from a mean of 15.41 to 25.97 seconds. The data were normally distributed, and no significant interactions between sides were identified. 


\section{Functional Movement Assessment Outcomes (DMA Analysis)}

Of the data collected from 37 participants who completed pre-test DMA movement analysis and the training intervention, data from only 28 of these participants were included in analysis of DMA measures due to an internal coding error beyond the scope of the research team's control. The data for 9 participants were therefore excluded from statistical analysis.

\section{DMA, Full Squat Test}

A paired samples t-test showed a significant difference in time $(p=0.019)$ when comparing pre-test to post-test values (Graph 3). The DMA full squat score improved from a mean of 42.31 points pre-training to 46.97 points posttraining. The data were normally distributed, and no significant interactions were identified.

\section{$D M A$, Step-Up Test.}

A mixed two-way analysis of variance (ANOVA) indicated a significant difference in time $(p=0.007)$, pre-test as compared to post-test (Graph 4$)$. Mean score improved from 17.55 at pre-test to 21.19 points post-test, comparing both sides collectively. Viewed unilaterally, the mean left step-up test improved from a score of 14.55 pre-training to 20.46 post-training, while mean right step-up score improved only marginally from a score of 20.55 pre-training to 21.94 post-training.

When testing the second null hypothesis examining the main effect of "side of body", (left versus right) a significant difference $(p=0.001)$ was found with the

DMA Full Squat Test, Pre- vs. Post-training Outcomes

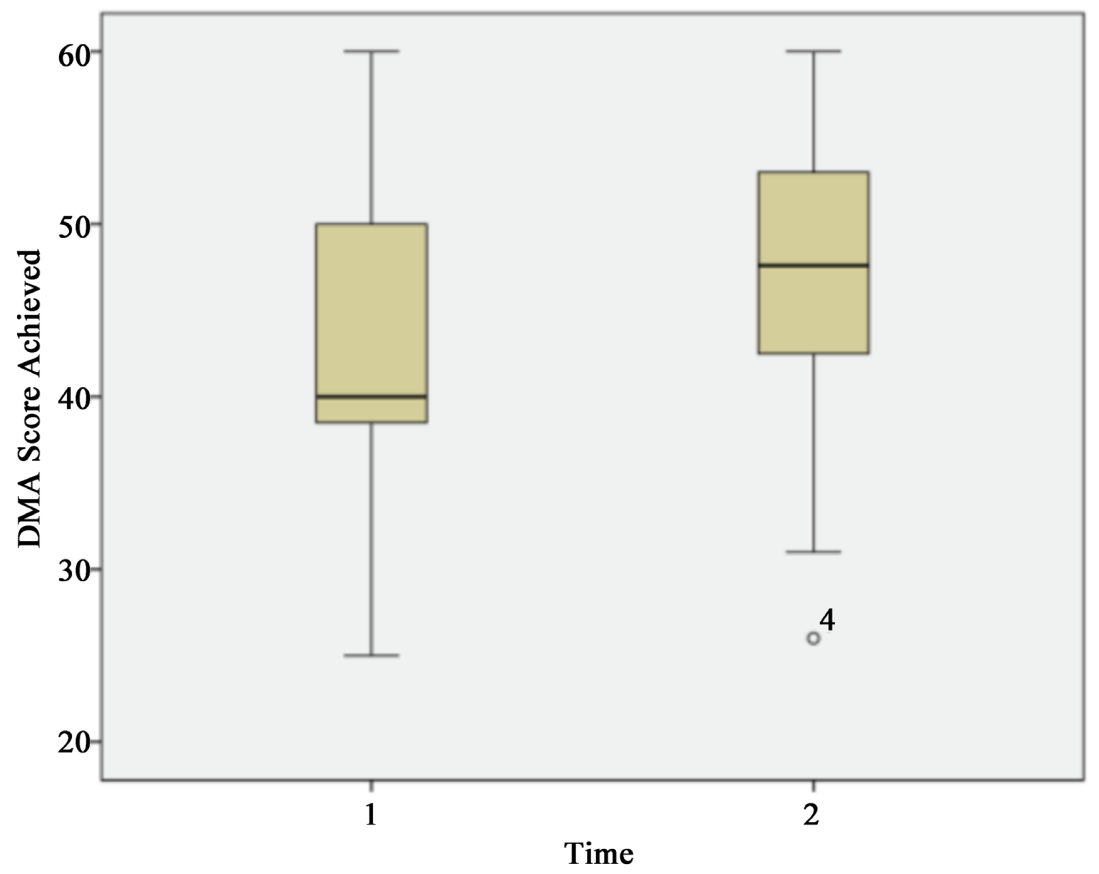

Graph 3. Dynamic Movement Assessment measures (score achieved) for full squat test depicted along vertical axis, across Time 1 (pre-test interval) and Time 2 (post-test interval) dimensions on the horizontal axis. 
DMA Step Up Test, Pre- vs. Post-training Outcomes

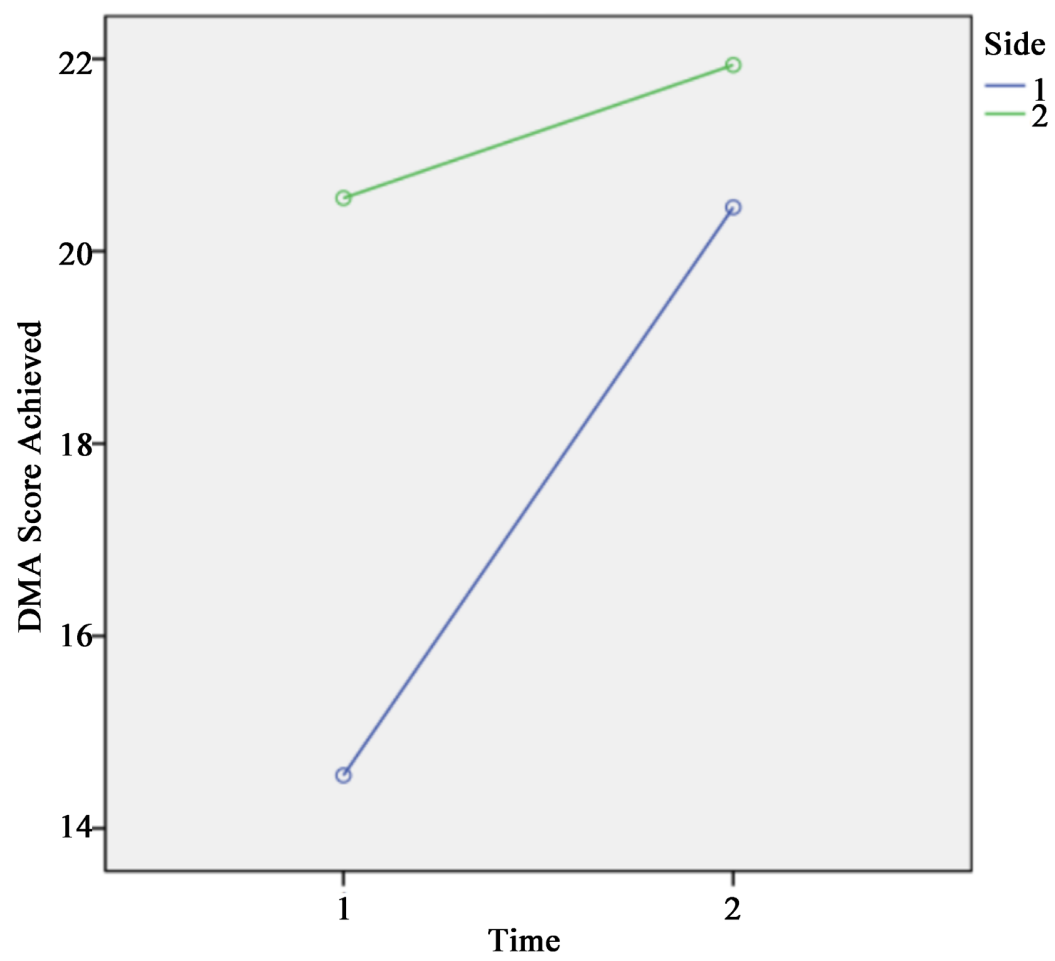

Graph 4. Dynamic Movement Assessment measures (score achieved) for the step-up test depicted along vertical axis, across Time 1 (pre-test interval) and Time 2 (post-test interval) dimensions on the horizontal axis. Left lower extremity shown as blue line, right lower extremity shown as green line.

mixed two-way ANOVA, demonstrating that the right side of the body exhibited higher scores than the left side of the body. Furthermore, the ANOVA indicated that there was a significant interaction between time and side of the body ( $p=$ 0.041). Post hoc comparisons further illuminated the specifics of these findings. All post hocs were completed with a paired samples t-test and a Bonferroni correction with a critical $p$-value of 0.0125 .

In a post-hoc comparison of pre-test left and pre-test right scores, the right side exhibited a significantly higher score $(p=0.000)$. There was also a significant difference in left step-up pre-test and left step-up post-test scores ( $p=$ 0.002). Post hoc analysis between the left step-up post-test and right step-up post-test indicated no significant differences $(p=0.424)$, and the post hoc analysis between right step-up pre-test and right step-up post-test indicated no significant differences $(p=0.363)$. The mean DMA score for the left step-up test improved from a score of 14.55 pre-training to 20.46 points post-training. However, the mean score for the right step-up test improved only marginally from a score of 20.55 to 21.94 points. The data for the right step-up pre-test are skewed, but despite the non-normal distribution, the decision was made to proceed with parametric procedures in our statistical analysis.

DMA, Single-Leg Squat Test

A mixed two-way ANOVA indicated a significant difference in time, pre-test 
and post-test $(p=0.000)$ with mean score improving from 15.81 at pre-test to 25.82 points at post-test, when considering bilateral lower extremities collectively (Graph 5). Specifically, the left single-leg squat improved from a mean score of 15.81 pre-training to 25.82 points post-training $(p=0.000)$, and right single-leg squat improved from a mean score of 18.25 to 27.52 points $(p=0.000)$.

These results reject our second null hypothesis, which originally stated there would be no significant difference in the main effect mean between sides of the body. The mixed two-way ANOVA described above indicates that there was a significant difference $(p=0.000)$ with the right side of the body scoring higher than the left side of the body. The third null hypothesis tested was that there would be no significant interaction between time and side of the body. The ANOVA did indeed confirm no significant interaction between these two variables, as described above.

\section{DMA, Single-Leg Hop Test}

A mixed two-way ANOVA showed a significant difference in time between pre-test and post-test scores of the DMA single-leg hop test (Graph 6). Left single-leg hop scores improved from a mean of 10.91 pre-training to 21.83 points post-training $(p=0.000)$, and right single-leg hop scores improved from a mean of 12.96 to 22.79 points $(p=0.000)$. The main effect pre-test mean was 16.37 compared to 17.79 points at post-test. When testing the second null hypothesis

DMA Single-Leg Squat Test, Pre- vs. Post-training Outcomes

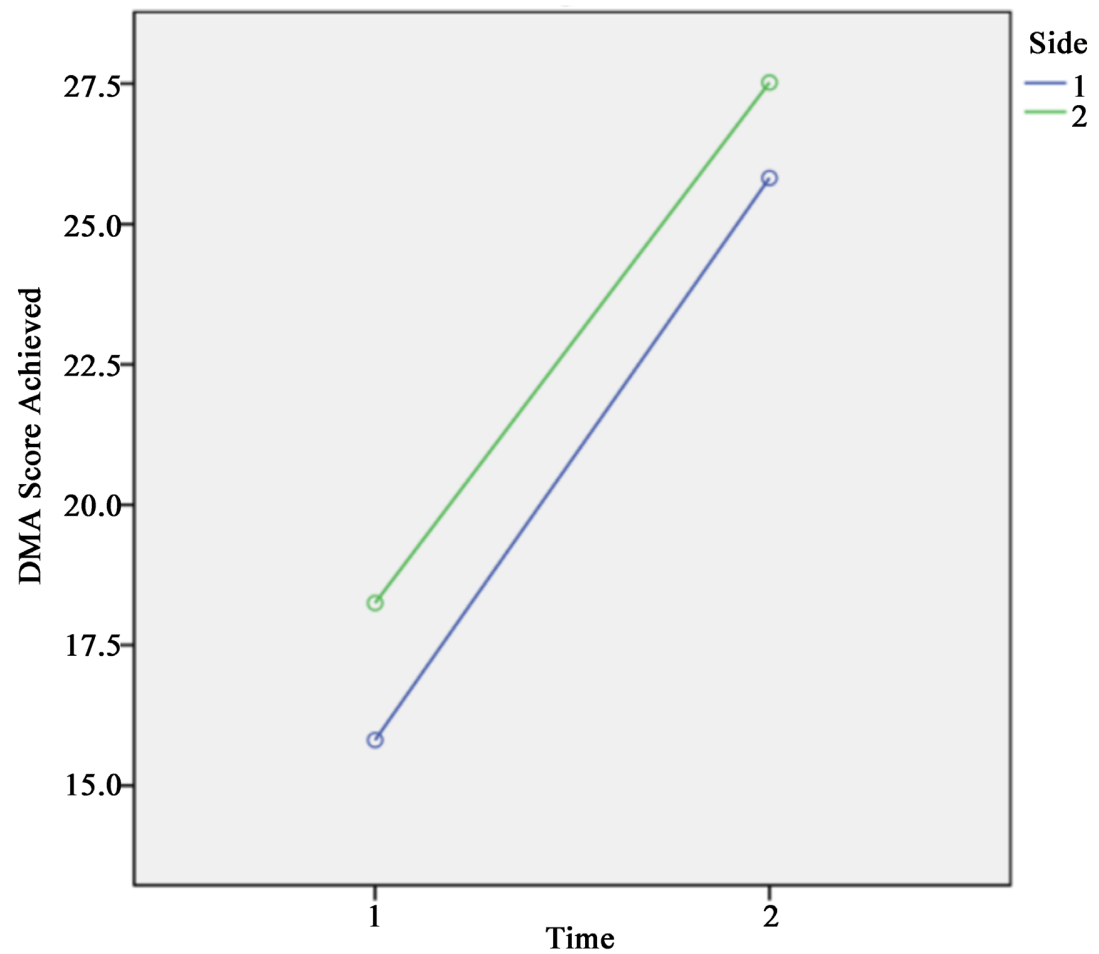

Graph 5. Dynamic Movement Assessment measures (score achieved) for the single-leg squat test depicted along vertical axis, across Time 1 (pre-test interval) and Time 2 (post-test interval) dimensions on the horizontal axis. Left lower extremity shown as blue line, right lower extremity shown as green line. 
DMA Single-Leg Hop Test, Pre- vs. Post-training Outcomes

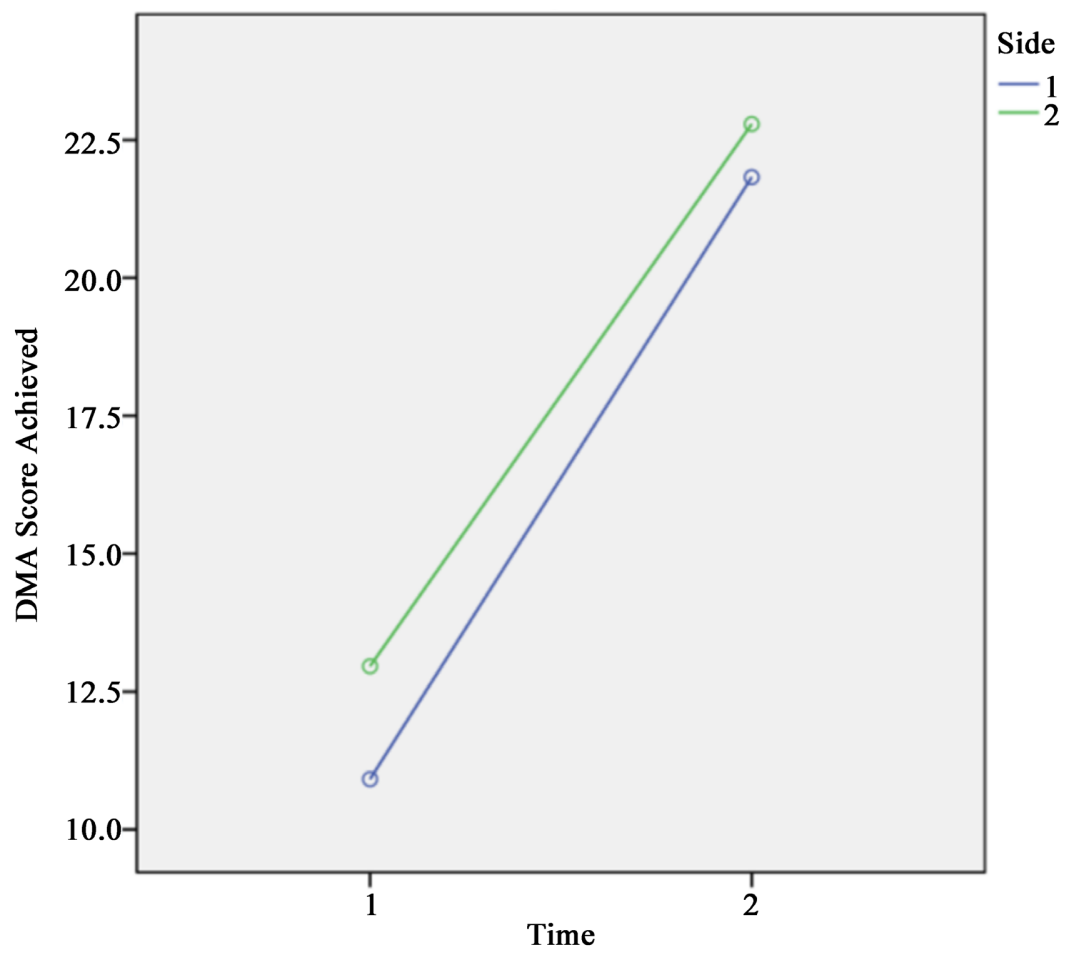

Graph 6. Dynamic Movement Assessment measures (score achieved) for the single-leg hop test depicted along vertical axis, across Time 1 (pre-test interval) and Time 2 (post-test interval) dimensions on the horizontal axis. Left lower extremity shown as blue line, right lower extremity shown as green line.

that there would be no difference between the side of the body (left vs. right), the ANOVA conveyed a significant difference $(p=0.007)$, indicating that the right side had a significantly higher score than the left side.

The third null hypothesis tested was that there was no significant interaction between time and side. The ANOVA outcomes illustrate that there was no significant interaction identified between side and time $(p=0.389)$, and no post-hoc analysis was necessary.

\section{Discussion}

During dynamic change of direction movements in the closed kinetic chain, female athletes display excessive valgus moments in the lower extremities with insufficient employment of stabilizing musculature, especially in that of the proximal hip architecture, making them subsequently more vulnerable to musculoskeletal trauma while participating in dynamic pivoting sports [16]. The results of the present investigation illustrate clear enhancements in the biomechanical profiles of these female participants, as evidenced by significant improvements in strength, dynamic balance, and performance in a functional movement analysis (3D-DMA) scores following an 8-week NMT intervention. These data suggest that the athletes who participated in the study will be at decreased risk for injury relative to their pre-training states of function for the duration in which the 


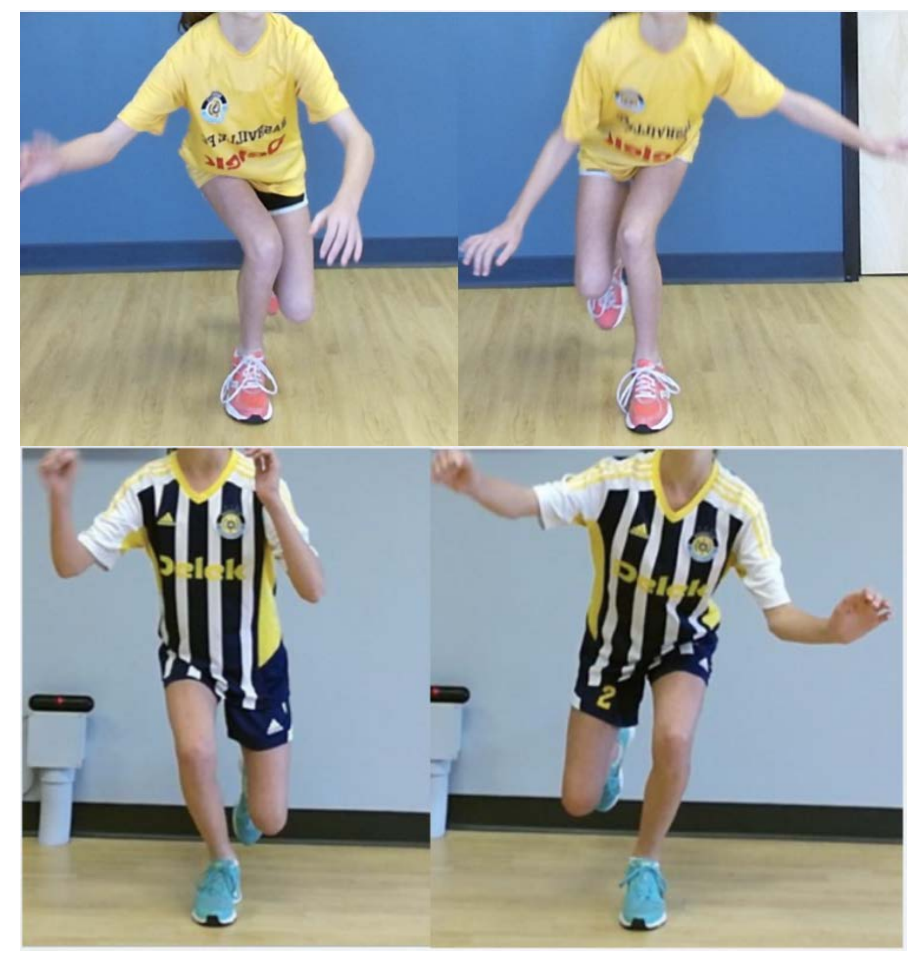

Image 3. DMA Single-Leg Hop Test, an example of positive outcomes illustrated in one of the participants following the intervention. Peak angles of joint range of motion excursion in each lower extremity depicted at pre-training interval (top) as compared to post-training (bottom).

intervention effects are maintained.

At pre-testing, the majority of the subjects presented with a constellation of suboptimal profiles including varus or valgus displacement and/or insufficient capacity to effectively move through a full, functional range of motion during their movement analyses. The improvement in 3D-DMA scores demonstrated in the movement assessments analyses indicate that athletes were able to move through a more complete range of motion while inhibiting high-risk, excessive frontal plane displacement during the loading tasks following the NMT intervention (participant example shown in Image 3). By decreasing the tendency for valgus loading moments and increasing mobility in non-threatening arcs of motion, risk for traumatic injury in the lower quarter are theoretically decreased [4]. In parallel with these observations, the subjects' improvements in proximal hip strength and dynamic balance collectively contribute towards the development and maintenance of more optimal movement strategies and a healthier biomechanical profile overall, supporting the inherent demands of training, competition, and performance.

A minor note of clarification with respect to the absence of significant differences yielded in the dynamic balance test in the eyes-open condition; these results were anticipated given the population tested and the imposed ceiling on time for this examination. The subjects were asked to stop after 30 seconds, which as expected was met at pre-testing by the majority of participants, indi- 
cating the subjects already had strong balance with their eyes open and, on average, did not have significant room to improve for this test within the parameters established. By contrast, for balance testing in the eyes-closed condition, significant differences were indeed observed between pre-test and post-test measures, indicating improvements in proprioception and vestibular function as they are associated with neuromuscular control. Interestingly, the examiners subjectively noted a considerable decrease in limb tortuosity and frontal plane knee displacement from pre-test to post-test, particularly in the eyes-closed testing condition, but these data were not quantified using the present methods and may be a subject of interest to future researchers.

An ethical consideration worthy of notice pertains to working with young female participants, and the present authors support the premise that all appropriate measures should be taken to ensure the safety and privacy of these individuals, including the gaining of assent and consent for treatment in coordination with each of their family or appropriate guardian(s), educating all parties on methods of intervention, and providing support conditions and supervision to ensure a safe and productive training environment.

With regards to statistical analyses, the data for the DMA Right Step-Up Test were skewed due to the fact that some participants scored very poorly and some scored perfectly. This skewness did not occur in the post-test group because the subjects who scored very poorly improved their score and those that scored perfectly had no room for improvement. A similar situation occurred with the DMA Single-Leg Hop Test, as the pre-test data were skewed for both the left and right limb. Again, the post-test data were not skewed due to improvement of the lower scores.

\section{Limitations of the Investigation and Future Research}

The study did encounter some factors that were out of the control of the researchers that could have affected the outcomes of the intervention. First, this program was implemented in the middle of the athletes' competitive season, meaning they were practicing and playing matches over the same period of the training intervention. Second, no reliable means was established to quantify the extent to which the participants completed their NMT home prescriptions independently, beyond each of the individual sessions of formal training employed in the study. Third, due to an internal coding error, the 3D-DMA software did not store the results of 9 of the participants, explaining the difference in number of subjects recorded between strength and balance (37) and movement analysis testing (28) which were statistically accounted for. The results of the current study could be stronger in power given a higher number of subjects. Further, on the final day of post-testing, many of the subjects had participated in extracurricular sport competition or other strenuous activities prior to testing. These activities include, but were not limited to, basketball, swimming, and recreational running. Completion of these activities may have induced a level of sub-acute fatigue that was present at testing, particularly in those that had participated in competitive events prior. The improvement in outcomes observed despite the 
likely presence of various amounts of fatigue in these individuals is promising, as it suggests the athletes were able to demonstrate improved functional outcomes with a degree of resilience in the face of preceding extracurricular activities.

Another point of consideration is the metrics by which strength measures were produced, indicating solely kilograms of force exerted against the dynamometry device. While these data are indeed informative in illustrating improvements in strength outcomes, a more effective illustration would be converting these measures into a percentage of the subjects' body weight. Research has successfully been conducted in the past utilizing this format, which would allow for more direct comparison of our findings to past research and subsequent generalizability. Finally, no longitudinal data were collected to determine if the athletes suffered any injuries after completion of the intervention to determine if the training was successful in injury reduction as compared to previous years, and prior data were not available within the club for this comparison. Similarly, it is unclear as to how long the training effects lasted following the intervention due to the absence of these observations. The athletes were tested 10 days after the NMT intervention was completed, offering a degree of insight into the durability of the training, but it would be even more valuable for the assessments to be repeated again after a longer post-training interval. The present investigators recommend that future studies look at the longitudinal outcomes of the athletes and repeat post-testing procedures at a range of later intervals, with both genders and a wider range of age groups. Future research that is directed at different configurations of training implementation design with respect to duration, total volume, and differing times in the calendar year (in-season vs. off-season) would provide further insight which could be utilized for enhancing the effectiveness of intervening to promote functional resilience and performance capacity with these populations.

\section{Conclusions}

The present investigation offers evidence suggesting that an in-season NMT intervention is an efficacious means for improving strength, dynamic balance, and behavioral patterns of functional movement in adolescent female soccer players, which may collectively reduce the risk of musculoskeletal trauma. Interventional outcomes indicating improvements in proximal hip strength, dynamic stability, and functional movement scores in the frontal and sagittal planes were analyzed before and after an 8-week training intervention. This information is important in the development of similar biomechanical studies and injury prevention programs and adds to the current body of knowledge in the field of neuromuscular training. The 3D-DMA software successfully identified high-risk movement behavior in the population studied and was effectively used to quantify the extent of improvement in biomechanics following the training intervention. The utilization of such motion analysis technologies for objective measurement of biomechanics during functional dynamic loading demands is highly valuable for 
quantifying high-risk movement behavior related to ACL and other musculoskeletal pathomechanics [23], and is a useful tool in determining the extent of progress made over time in interventional sports medicine environments. Such pathways for assessment and interventional design are especially desirable amidst the inherent demands of the adolescent growth period, wherein the highest degree of emergent pathological movement behavior is often identified and potentially the greatest opportunity for healthy development exists as one builds the neural and musculoskeletal scaffolding to support the increased demands of adult competition.

The findings of this study support the premise that neuromuscular risk factors related to musculoskeletal trauma are quantifiable and can be positively influenced with specific training interventions. Further, these findings demonstrate the feasibility of implementing training interventions concurrently with in-season training and competition in youth female athletes, which is generally of greater complexity relative to off-season training designs due to the risks associated with excessive volume in work load for any given period. There is a clear need for the implementation of valid and reliable assessment methodologies which can produce accurate data describing movement behavior in multiple planes of motion. Such technology can both help to identify individuals at risk for injury, and be used as an objective lens through which to measure progress.

Future researchers would benefit from examining similar NMT interventions with larger sample size, incorporating athletes of different ages and genders. The use of intelligent motion analysis has the potential to substantially attenuate the alarming rates of musculoskeletal trauma currently identified in pivoting athletes, which could be invaluable in advancing the fields of sports medicine and rehabilitation. The generalizability of the present findings is limited to the subject population of athletic females between the ages of 10 and 15, and more research is warranted to determine the extent of improvement that may be imparted with such training in similar populations differing in age and gender. Future research is warranted to determine the durability of improvements elicited, quantify the potency of individual training activities, establish a minimum effective dose of various interventional strategies, employ parameters to establish the degree of later success achieved post-training, and explore the potential of protecting against of fatigue-inducing factors as they relate to movement profiles.

\section{Conflicts of Interest}

The authors declare no conflicts of interest.

\section{References}

[1] Kiapour, A.M., Kiapour, A., Goel, V.K., et al. (2015) Uni-Directional Coupling between Tibiofemoral Frontal and Axial Plane Rotation Supports Valgus Collapse Mechanism of ACL Injury. Journal of Biomechanics, 48, 1745-1751.

https://doi.org/10.1016/j.jbiomech.2015.05.017 
[2] Bien, D.P. (2011) Rationale and Implementation of Anterior Cruciate Ligament Injury Prevention Warm-Up Programs in Female Athletes. Journal of Strength and Conditioning Research, 25, 271-285. https://doi.org/10.1519/JSC.0b013e3181fb4a5a

[3] Beynnon, B.D., Vacek, P.M., Newell, M.K., et al. (2014) The Effects of Level of Competition, Sport, and Sex on the Incidence of First-Time Noncontact Anterior Cruciate Ligament Injury. The American Journal of Sports Medicine, 42, 1806-1812. https://doi.org/10.1177/0363546514540862

[4] Mather, R.C., Koenig, L., Kocher, M., et al. (2013) Societal and Economic Impact of Anterior Cruciate Ligament Tears. The Journal of Bone and Joint Surgery, 95, 17511759. https://doi.org/10.2106/JBJS.L.01705

[5] Herzog, M.M., Marshall, S.W., Lund, J.L., Pate, V., Mack, C.D. and Spang, J.T. (2017) Incidence of Anterior Cruciate Ligament Reconstruction among Adolescent Females in the United States, 2002 through 2014. JAMA Pediatrics, 171, 808-810. https://doi.org/10.1001/jamapediatrics.2017.0740

[6] Crichlow, R.J., Andres, P.L., Morrison, S.M., Haley, S.M. and Vrahas, M.S. (2006) Depression in Orthopaedic Trauma Patients. Prevalence and Severity. The Journal of Bone and Joint Surgery, 88, 1927-1933.

[7] Garcia, G.H., Wu, H.H., Park, M.J., et al. (2016) Depression Symptomatology and Anterior Cruciate Ligament Injury: Incidence and Effect on Functional Outcome-A Prospective Cohort Study. The American Journal of Sports Medicine, 44, 572-579. https://doi.org/10.1177/0363546515612466

[8] Wu, H.H., Liu, M., Dines, J.S., Kelly, J.D. and Garcia, G.H. (2016) Depression and Psychiatric Disease Associated with Outcomes after Anterior Cruciate Ligament Reconstruction. World Journal of Orthopedics, 7, 709-717. https://doi.org/10.5312/wjo.v7.i11.709

[9] Brophy, R.H., Schmitz, L., Wright, R.W., et al. (2012) Return to Play and Future ACL Injury Risk after ACL Reconstruction in Soccer Athletes from the Multicenter Orthopaedic Outcomes Network (MOON) Group. The American Journal of Sports Medicine, 40, 2517-2522. https://doi.org/10.1177/0363546512459476

[10] Neumann, D.N. (2010) Kinesiology of the Musculoskeletal System: Foundations for Rehabilitation. 2nd Edition, Mosby Incorporated, St. Louis, MS, 533-534.

[11] Ardern, C.L., Osterberg, A., Tagesson, S., Gauffin, H., Webster, K.E. and Kvist, J. (2014) The Impact of Psychological Readiness to Return to Sport and Recreational Activities after Anterior Cruciate Ligament Reconstruction. British Journal of Sports Medicine, 48, 1613-1619. https://doi.org/10.1136/bjsports-2014-093842

[12] Wiggins, A.J., Grandhi, R.K., Schneider, D.K., et al. (2016) Risk of Secondary Injury in Younger Athletes after Anterior Cruciate Ligament Reconstruction: A Systematic Review and Meta-Analysis. The American Journal of Sports Medicine, 44, 1861-1876. https://doi.org/10.1177/0363546515621554

[13] Shimokochi, Y. and Shultz, S.J. (2008) Mechanisms of Noncontact Anterior Cruciate Ligament Injury. Journal of Athletic Training, 43, 396-408.

https://doi.org/10.4085/1062-6050-43.4.396

[14] Pfeifer, C.E., Beattie, P.F., Sacko, R.S. and Hand, A. (2018) Risk Factors Associated with Non-Contact Anterior Cruciate Ligament Injury: A Systematic Review. International Journal of Sports Physical Therapy, 13, 575-587.

[15] Sugimoto, D., Alentorn-Geli, E., Mendiguchia, J., Samuelsson, K., Karlsson, J. and Myer, G.D. (2015) Biomechanical and Neuromuscular Characteristics of Male Athletes: Implications for the Development of Anterior Cruciate Ligament Injury Prevention Programs. Sports Medicine, 45, 809-822. 
https://doi.org/10.1007/s40279-015-0311-1

[16] Pollard, C.D., Sigward, S.M. and Powers, C.M. (2010) Limited Hip and Knee Flexion during Landing Is Associated with Increased Frontal Plane Knee Motion and Moments. Clinical Biomechanics, 25, 142-146. https://doi.org/10.1016/j.clinbiomech.2009.10.005

[17] Nielson, D.B. and Daugaard, M. (2008) Comparison of Angular Measurements by 2D and 3D Gait Analysis. Jönköping Univerity, Jönköping.

[18] Sigward, S.M. and Powers, C.M. (2006) The Influence of Gender on Knee Kinematics, Kinetics and Muscle Activation Patterns during Side-Step Cutting. Clinical Biomechanics, 21, 41-48.

[19] Silvers, H.J. and Mandelbaum, B.R. (2007) Prevention of Anterior Cruciate Ligament Injury in the Female Athlete. British Journal of Sports Medicine, 41, i52-i59. https://doi.org/10.1136/bjsm.2007.037200

[20] Dingenen, B. and Gokeler, A. (2017) Optimization of the Return-to-Sport Paradigm after Anterior Cruciate Ligament Reconstruction: A Critical Step Back to Move Forward. Sports Medicine, 47, 1487-1500. https://doi.org/10.1007/s40279-017-0674-6

[21] Lafayette Instrument (2015) Lafayette Manual Muscle Testing System. Human Evaluation from Lafayette Instrument Company.

http://www.lafayetteevaluation.com/product_detail.asp?itemid=26

[22] DETECTO (2015) Weigh Beam Eye-Level.

https://www.detecto.com/product/product-overview/Physician-Scales/Weigh-Beam -Eye-Level

[23] Stone, E.E., Butlar, M., McRuer, A., Gray, A., Marks, J. and Skubic, M. (2013) Evaluation of the Microsoft Kinect for Screening ACL Injury. Annual International Conference of the IEEE Engineering in Medicine and Biology Society, Osaka, 3-7 July 2013, 4152-4155.

[24] Weber, I., Koch, J., Mekemper, J., Friedl, K. and Hartmann, U. (2012) Is the MS Kinect Suitable for Motion Analysis? Biomedizinische Technik, 57, 664. https://doi.org/10.1515/bmt-2012-4452 